\title{
Quelques remarques sur les cadences dans la musique de vénerie française
}

Some ideas on the cadenzae in French venery music

\section{Luc Breton}

\section{(2) OpenEdition}

Journals

Édition électronique

URL : http://journals.openedition.org/ethnomusicologie/1406

ISSN : 2235-7688

Éditeur

ADEM - Ateliers d'ethnomusicologie

Édition imprimée

Date de publication : 31 décembre 1994

Pagination : 175-190

ISBN : 2-8257-0503-9

ISSN : 1662-372X

\section{Référence électronique}

Luc Breton, «Quelques remarques sur les cadences dans la musique de vénerie française », Cahiers d'ethnomusicologie [En ligne], 7| 1994, mis en ligne le 03 janvier 2012, consulté le 19 avril 2019. URL: http://journals.openedition.org/ethnomusicologie/1406

Ce document a été généré automatiquement le 19 avril 2019.

Tous droits réservés 


\title{
Quelques remarques sur les cadences dans la musique de vénerie française
}

Some ideas on the cadenzae in French venery music

\author{
Luc Breton
}

\section{Avant propos}

1 L'esprit moderne, imprégné de notions évolutionnistes, a souvent du mal à concevoir que les pratiques d'une époque puissent trouver leur justification dans des doctrines qu'il pense appartenir exclusivement à un passé reculé.

2 C'est ainsi que les changements intervenus dans l'esprit occidental à la fin du XVIII siècle et au début du XIX nous font refuser une certaine pérennité à nos traditions, que nous accordons curieusement bien volontiers à d'autres civilisations.

3 Selon l'ancienne optique, l'histoire, dans sa globalité, n'était qu'une illustration dans le monde sensible de la Vérité immanente, exposée par la doctrine chrétienne. Or la Vérité, étant un plérôme, revêt des aspects paradoxaux et ne s'exprime que de manière contradictoire (apophatique et cataphatique). Chaque époque ayant, selon la sagesse populaire, sa vérité, si un aspect de la doctrine ne peut pas coexister à un moment donné avec son opposé, comme le lumineux avec l'obscur, ces deux aspects pourront se succéder dans le cours de l'histoire sans contradiction véritable, car dans des contextes différents.

Une pratique telle que la chasse à courre doit se regarder avec ces yeux-là si l'on veut y trouver un intérêt. La vénerie française n'est alors rien d'autre qu'une liturgie par laquelle la doctrine prend corps, rendue ainsi plus intelligible pour une catégorie de personnes particulières. Il s'agit d'une action rituelle dans laquelle les comportements sont en quelque sorte stéréotypés, et chaque événement survenu au cours de la chasse est alors considéré par le veneur comme une intervention providentielle qui le concerne personnellement tout comme elle concerne le groupe auquel il appartient. 
Ceci est comparable aux aventures des quêteurs dans la Quête du Graal de Chrétien de Troye, aventures qui sont révélatrices d'un état spirituel.

C'est à la partie liturgique de la chasse qu'appartient le sujet qui nous occupe. Les fanfares de la vénerie actuelle, comme nous tentons de le montrer, indiquent au veneur le point de vue qui doit être le sien sur chaque action exigée par le déroulement de la chasse.

Il est évident que les sonneries sur lesquelles nous allons nous pencher sont spécifiques $\mathrm{du} \mathrm{XVII}^{\mathrm{e}}$ et XVIII ${ }^{\mathrm{e}}$ siècles, et demeurent d'ailleurs quasi inchangées depuis. Mais elles ont été précédées d'autres sonneries, comme les cornures, fanfares typiques du cor ou olifant à deux tons, lesquelles fanfares, comme nous le voyons chez Gaston Phoebus, avaient au fond la même structure que celle qui fut adoptée pour la trompe à douze sons.

Plusieurs des fanfares de trompe sont attribuées au Marquis de Dampierre, maître de la vénerie de Louis XV, mais beaucoup lui sont certainement antérieures, comme les Honneurs, la St-Hubert ou la tête bizarde (air de « Il est né le divin enfant ») qui ont été empruntées à la trompe d'église pour la plupart.

En ce qui concerne la structure de ces fanfares, des cornures ou même de certaines sonneries militaires encore en vigueur sous Napoléon Bonaparte, c'est chez saint Augustin qu'il faut trouver l'explication d'une pratique actuelle quoiqu'en voie de disparition. Depuis le début du XVIII ${ }^{\mathrm{e}}$ siècle, nous nous sommes progressivement habitués à ce que l'argument artistique, tant dans le domaine musical que dans les arts plastiques, soit futile ou sans portée doctrinale. Il est difficile de trouver, aussi bien dans l'Antiquité tardive que dans la période médiévale, voire celle de la Renaissance, une représentation de scène purement pittoresque : le sujet eût été considéré comme dépourvu d'intérêt.

- Par contre, le XVII ${ }^{e}$ siècle finissant et le XVIII ${ }^{e}$ raffolèrent des sujets moraux ou des scènes de genre (Diderot, encensant L'Accordée de village de Greuze et autres tableaux moralisateurs et touchants, passe alors pour un esprit profond). La chose n'a fait qu'empirer depuis, jusqu'à la disparition de l'art figuratif, ce qui simplifiait le travail des artistes, puisqu'on n'exigeait plus d'eux aucune connaissance doctrinale ou culturelle, mais un talent «brut ». En revanche, l'esthétique demeurait souvent liée à une doctrine que l'on prétendait rejeter.

1 Lorsqu'on examine les pratiques artistiques traditionnelles, il faut constater qu'il en va tout autrement, y compris dans le choix esthétique. Sous cet angle de vue, la musique de vénerie française est très remarquable. En effet, dans « l'art du bien-sonner de la trompe de chasse ", aucun détail n'est vide de symbolisme au sens le plus fort du terme.

Il est impossible, dans le cadre de cet article, de traiter les nombreux points qui illustreraient cette affirmation, mais l'examen de la seule structure des fanfares sonnées en vénerie française et leur rapport avec la doctrine catholique permettront de s'en faire une idée.

\section{Les cinq types de fanfare}

La vénerie française, qui a évolué dans le détail au cours des siècles, a conservé une tradition presque intacte et utilise cinq types de sonneries dont certaines sont bien fixées, d'autres ont subi de légères variantes, alors que celles qui sont par nature vouées au renouvellement continuent de se composer conformément à l'ancienne manière : c'est donc un art bien vivant. 
Les cinq catégories sont :

- Les tons d'animaux qui sont pratiquement fixés.

- Les fanfares de circonstances, également codifiées.

- Les fanfares de personnes et d'équipages, transmissibles, renouvelables ou composées selon les besoins.

- Les fanfares de lieux telles que Souvenirs et Echos qui constituent une catégorie très souple.

- Les Messes de St Hubert qui forment un groupe central tant du point de vue du style que par les moyens employés (sons bouchés, radoucis en grand nombre, carillons, etc.).

Il existe aussi des «bien-allés » et " tons pour chiens » qui ne sont cependant ni codifiés ni répertoriés mais se transmettent soigneusement d'un piqueur à l'autre.

Dans ces quelques pages, nous nous limiterons à expliciter la structure la plus commune des fanfares des quatre premiers groupes.

\section{Structure générale des fanfares}

17 Prenons comme exemple le "Vol-ce-l'est " ( = vois, c'est lui), fanfare qui signale aux autres veneurs que l'empreinte du pied de l'animal que l'on courre a été reconnue sans équivoque :

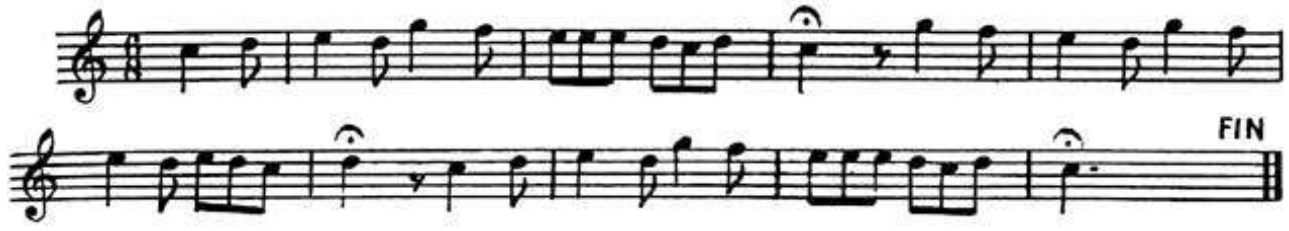

Cette fanfare transmise, arrangée ou composée, on ne sait, par le marquis de Dampierre, maître d'équipage du roi Louis XV, est parfaitement symétrique : elle commence par une phrase conclue par une cadence sur la tonique, suivie d'une seconde phrase avec cadence sur le second degré, puis finit avec une troisième phrase identique à la première.

C'est là le genre le plus simple et le plus fréquent. Il en va de même pour cette autre fanfare, «L'arrivée au rendez-vous ».

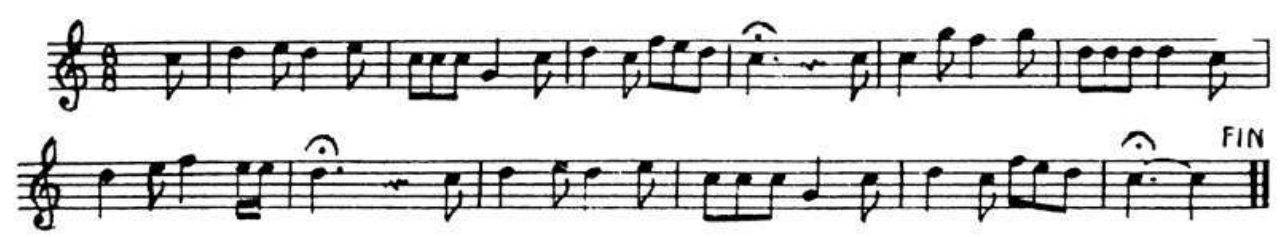

Ici encore : une cadence sur la tonique do, une cadence sur ré, second degré, une cadence sur do.

21 Pour entrevoir l'importance des cadences, il convient maintenant de s'éloigner du domaine purement musical, pour demander des éclaircissements à la doctrine et à la théorie physique des sons.

\section{La périchorèse}

Dans le cadre du christianisme, le monde est considéré comme créé selon un processus qui lui confère liberté et justification, et qui est en quelque sorte calqué sur ce qui se passe à l'intérieur de la Trinité elle-même. Ce processus, qui ne doit pas être assimilé à 
quoi que ce soit de systématique, a été nommé périchorèse par les Pères de l'Eglise grecs et circumincessio par les latins. De la périchorèse trinitaire, nous ne savons guère plus que ce que dit le Christ lui-même «Mon Père est en moi tout comme je suis dans le sein du Père » (Jn 10, 28-38 et aussi Jn 1, 10,15) et ceci est vrai des trois personnes de la Trinité les unes par rapport aux autres.

Par contre, dans le monde créé, il y a une forme de périchorèse qui nous est beaucoup plus accessible.

Le Verbe Créateur crée les êtres, c'est la génésis - création - alors que dans la Trinité il y a engendrement. L'être créé a deux possibilités, aussitôt après sa création, dès qu'il prend conscience de lui-même : ou bien il reste sur cette vision égocentrique, et c'est la chute, ou bien il rapporte son existence à sa Cause : c'est l'épistrophè - conversion - dans un élan volontaire et conscient. Ce cycle peut se résumer dans le schéma suivant :

\title{
CRÉATEUR
}

\section{génésis generation (création)}
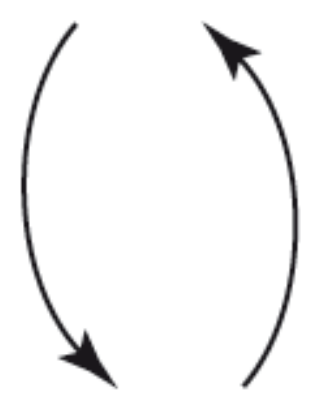

\author{
epistrophè \\ conversio \\ (conversion)
}

\section{ÊTRE CRÉÉ}

Il s'agit donc d'un élan réciproque entre deux êtres, l'un accordant le don de participation à sa vie à l'autre, qui, alors, se retourne dans un mouvement de conversion vers sa cause. L'aspect le plus connu de la périchorèse est l'amour, qui, avant tout, n'est pas un sentiment.

Si sentiment il y a, c'est bien la conséquence de l'accomplissement non entravé de la périchorèse, tant au sein de la Trinité elle-même, où elle revêt un aspect très mystérieux, qu'entre le Créateur et sa créature, ou même parmi les créatures entre elles.

Or, l'enjeu de la cadence en musique n'est rien d'autre que le symbole (entendu comme ce qui porte en soi) de la périchorèse. Partant de notes qui, implicitement, définissent une tonique (ici les harmoniques naturels de la tonalité de la trompe) on retombe sur cette tonique en fin de phrase (cadence de tonique = conversion) ou à côté (cadence de ré équivalente ici à un certain type de chute, mais non définitive comme nous le verrons plus loin). Il n'est pas excessif, dès lors, de dire que l'examen des cadences en musique est d'une certaine manière un problème de vie ou de mort, tout ceci étant naturellement simplifié à l'extrême. 


\section{L'ange sonneur de trompe} Litteram, aux chapitres XXII à XXV du livre quatrième (voir texte intégral en annexe). La fonction musicale particulière des anges, celle qu'ils exercent en rapport avec leur nature même, est exposée ainsi par St Augustin : dans la Genèse, le premier jour a été créée la Lumière. Or les luminaires, soleil et lune, ne l'ont été que le quatrième jour. Il s'agit donc, dit St Augustin, non de la lumière matérielle, mais d'une Lumière Spirituelle, en l'occurrence, les Anges.

31 Aussitôt après cette création, il est écrit : «il y eut un soir, il y eut un matin ». Etrange affirmation, si l'on songe qu'il ne peut guère y avoir de soir qui ne soit précédé d'un matin. La raison en est donc qu'aussitôt créés, ces anges prennent conscience d'euxmêmes pour eux-mêmes, en tant qu'ils ne sont pas ce que Dieu est. Une telle vision est repliée sur elle-même, sans éclairage divin : c'est une vision du soir dite vespérale. Les anges ainsi créés ont donc la possibilité d'en rester là, c'est le cas des anges déchus qui ferment les yeux (fig. 1) : il se complaisent en eux-mêmes, et c'est la chute. Les autres par contre, après s'être considérés, rapportent leur existence au Créateur et tournent leurs regards vers Lui, la véritable Lumière incréé : ils ont une vision du matin dite matutinale. Voici donc les anges doués a priori de deux types de visions : celle des êtres et des choses tels qu'ils sont dans la lumière du monde créé, et celle qu'ils offrent à la Lumière divine incréée dans leur véritable causalité voulue par le Verbe lui-même. Les anges verront tout selon ces deux modes tout au long des six jours de la création et continueront par la suite : c'est là leur ministère. 
Fig. 1 : « La Chute des Anges » dans Les très riches heures du Duc de Berry

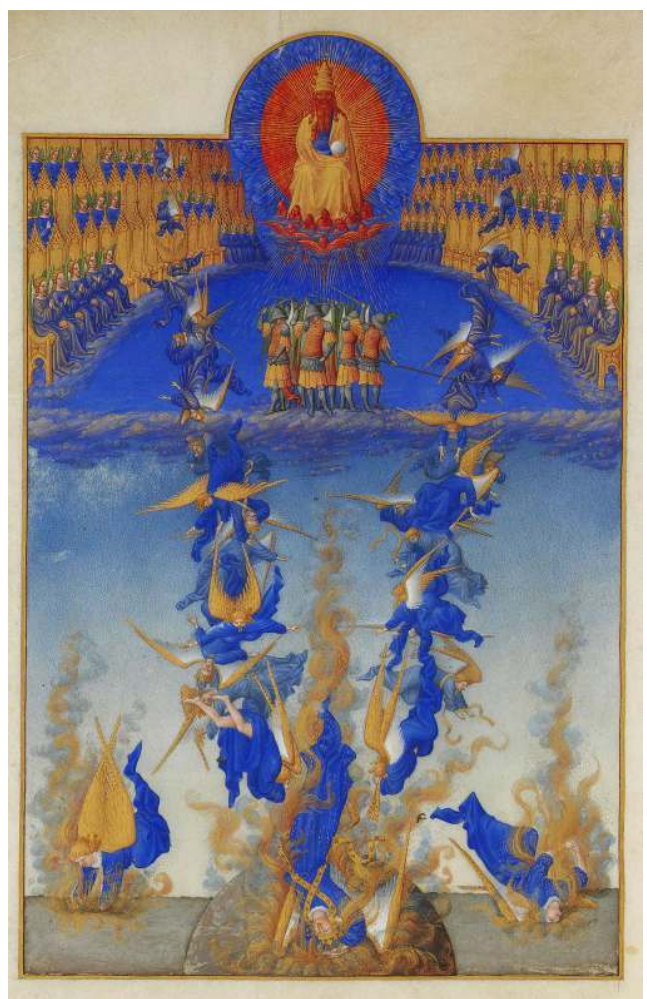

Bibliothèque Nationale, Paris.

33 Mais dès le second jour de la création, après la leur propre, sont créés d'autres êtres. Ils sont préalablement portés à la connaissance des anges quand il est dit « et il en fut ainsi ». Cela signifie qu'avant que les êtres créés existent, leur nécessité voulue par le Créateur est d'abord connue des anges qui en ont alors une vision dite diurnale. 
Fig. 2 : Trompe dite d'Orléans (enroulée à trois tours et demi).

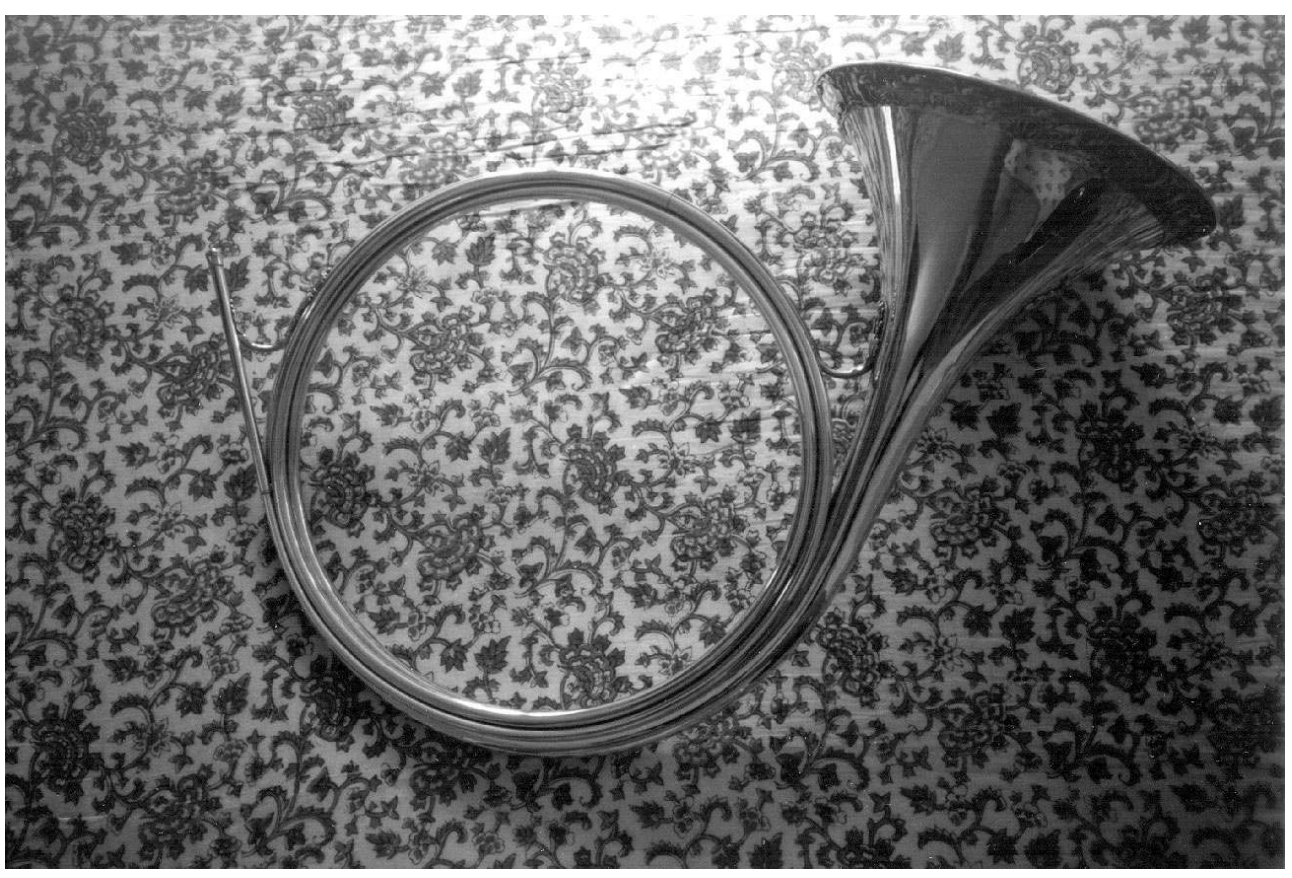

Or ces trois visions angéliques correspondent aux trois phrases ou trois groupes de deux phrases qui constituent les sonneries qui nous occupent. La première phrase se rapporte à la vision diurnale: prise de connaissance par le sonneur de la volonté divine. La seconde, une fois la chose connue dans le Principe, est prise de connaissance de la chose pour elle-même, c'est une connaissance vespérale. Enfin la troisième se rapporte à la connaissance matutinale qui justifie aux yeux du Créateur la chose ou l'événement existant par cette offrande qu'est l'épistrophè ou conversion.

Il est dès lors possible d'entrevoir l'importance de la cadence de chaque phrase, si l'on sait que la note cadencielle représente l'être créé ou incréé auquel est rapportée la vision en question. Les notes produites par la trompe, excepté ce qu'on appelle les sons bouchés, sont des harmoniques de la fondamentale ou tonique. Cela signifie qu'elles ont une fréquence qui se trouve dans un rapport numérique simple ou fractionnaire par rapport à cette même tonique.

Il convient donc de donner cette séquence des harmoniques naturels et sa signification.

\section{La séquence des harmoniques naturels et leur interprétation}

La séquence des harmoniques produits par une colonne d'air vibrante est la même que celle d'une corde vibrante qui était l'étalon tonal des chanteurs médiévaux (monocorde régulateur) à une exception près, pour la trompe, qui est l'absence de l'Ut.

38 La note la plus basse de la trompe est donc le $\mathrm{Do}_{2}$ dit Do de pédale, qui ne se vibre pas pour qu'il soit conservé dans toute sa pureté.

39 Cette séquence peut, pour les quatre octaves produites par la trompe, se représenter ainsi : 


\begin{tabular}{|l|l|l|l|l|l|l|l|l|}
\hline $1^{\mathrm{er}}$ octave & $\left(\mathrm{Ut}_{1}\right)$ & & & & & & & $\mathrm{do}_{2}$ \\
\hline $2^{\mathrm{e}}$ octave & $\mathrm{do}_{2}$ & & & & $\mathrm{sol}_{3}$ & & & $\mathrm{do}_{4}$ \\
\hline $3^{\mathrm{e}}$ octave & $\mathrm{do}_{4}$ & & $\mathrm{mi}_{5}$ & & $\mathrm{sol}_{6}$ & & $\mathrm{si}^{+}{ }_{7}$ & $\mathrm{do}_{8}$ \\
\hline \hline $4^{\mathrm{e}}$ octave & $\mathrm{do}_{8}$ & $\mathrm{ré}_{9}$ & $\mathrm{mi}_{10}$ & $\mathrm{fa}^{+}$ & $\mathrm{sol}_{12}$ & $\mathrm{la}^{-}$ & $\left(\mathrm{sib}_{13}^{+} \mathrm{si}^{-}{ }_{15}\right)$ & $\mathrm{do}_{16}$ \\
& & & & & & & & \\
\hline
\end{tabular}

\section{Remarques}

- L'indice indique le rang de l'harmonique. S'il s'agissait d'une corde, ce serait une corde qui vibrerait en $1,2,3 \ldots$ fuseaux.

- Pour mémoriser facilement l'apparition des harmoniques au fil des octaves, il suffit de remarquer que d'une octave à la suivante s'intercalent des notes intermédiaires supplémentaires par rapport à l'octave précédente.

- La première ligne définit l'octave juste, la seconde la quinte juste (harmonique) et son renversement (l'intervalle sol-do) la quarte juste. La quinte de la tonique est dite dominante (de Dominus, le Seigneur), car cet intervalle permet par empilement de générer tous les tempéraments autres que le tempérament égal. L'octave introduit dans la séquence ininterrompue des fréquences possibles un repère périodique indispensable à la musique instrumentale.

- Tous les intervalles harmoniquement justes se calculent à l'aide de ce tableau. Ainsi, par exemple, se calcule la fréquence de la sous-dominante par rapport à la tonique virtuelle Ut.

Pour connaître le rapport de fréquence entre deux sons, il suffit de diviser le nombre correspondant au rang d'harmonique de la note la plus haute par celui de la note la plus basse :

- Rapport de fréquence entre $\mathrm{Ut}_{1}$ et $\mathrm{do}_{2}=2 / 1=2$

- Rapport de fréquence entre $\mathrm{sol}_{3}$ et $\mathrm{do}_{2}=3 / 2$

- Renversement de l'intervalle $\left[\mathrm{do}_{2}-\mathrm{sol}_{3}\right]=2 / 3=\left[\mathrm{fa}_{2 / 3} \mathrm{do}_{2}\right]$

- En ramenant le $\mathrm{fa}_{2 / 3}$ ainsi obtenu dans l'intervalle $\mathrm{do}_{2}-\mathrm{sol}_{3}$, on doit doubler la fréquence, donc prendre son octave qui sera dans le rapport $4 / 3$ avec $\left(\mathrm{Ut}_{1}\right)$.

- L'intervalle $\left[\mathrm{fa}_{4 / 3}-\right.$ sol $\left._{3}\right]$ est donné par $3 / 2: 4 / 3=9 / 8$ identique à l'intervalle $\left[\mathrm{do}_{8}-\right.$ ré $\left._{9}\right]$ seconde majeure.

41 Ces calculs peuvent se résumer dans le schéma suivant :

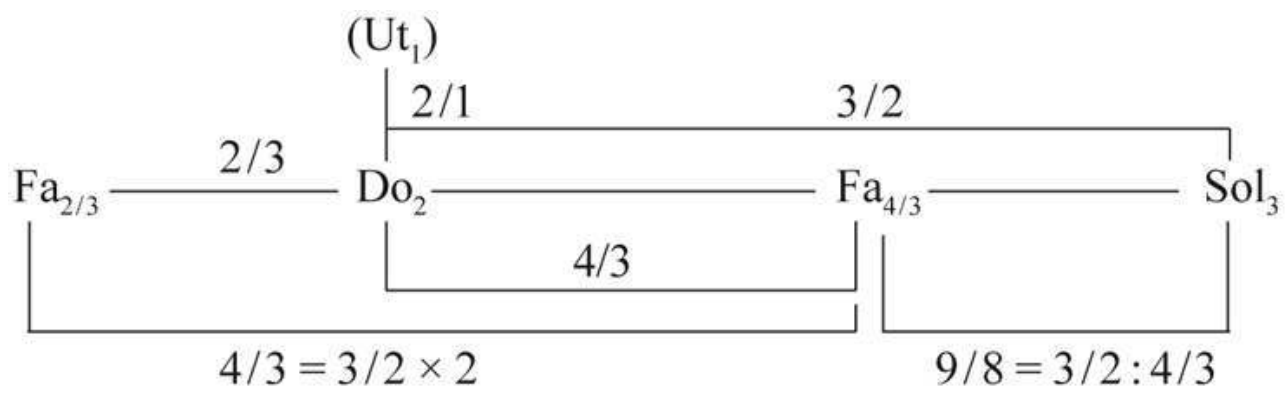



nombre gouvernant l'harmonique. C'est ainsi que le nombre, en musique, est traditionnellement considéré non comme une quantité (par exemple la mesure de la fréquence en hertz) mais comme la nature véritable d'un être sonore, qui lui-même rend présent dans la musique, un être incréé ou créé.

Si l'Ut $t_{1}$ représente l'Un ineffable et non manifesté, le do (associé également à Dominus, le Maître, d'un autre point de vue selon lequel on dit de la note sol qu'elle est dominante) n'est ni tout à fait le même son, ni tout à fait un autre. Ce do est ce dont Jean Scot Erigène dans le De divisione Naturae (III 683 a) dit: «Entreprenant de descendre de la suressentialité de sa nature (Ut) selon laquelle on l'appelle Non-Etre dans ses causes primordiales, Dieu se crée Lui-même et se fait principe (do)... »

\section{Avec l'harmonique 3 (sol associé à Sol Justitiae, Soleil de justice en parlant du Fils, Verbe} créateur) apparait un intervalle qui va engendrer toutes les notes du tempérament naturel. La note fa (femina) obtenue par renversement de l'intervalle de quinte est associée à la Vierge Marie, la «mulier amicta sole » de l'Apocalypse, comme le montre la peinture de Gentile da Fabriano (fig. 3). Il en est de même des harmoniques suivants qui correspondent tous à des êtres créés bien définis.

Fig. 3 : Triptyque de Gentile da Fabriano.

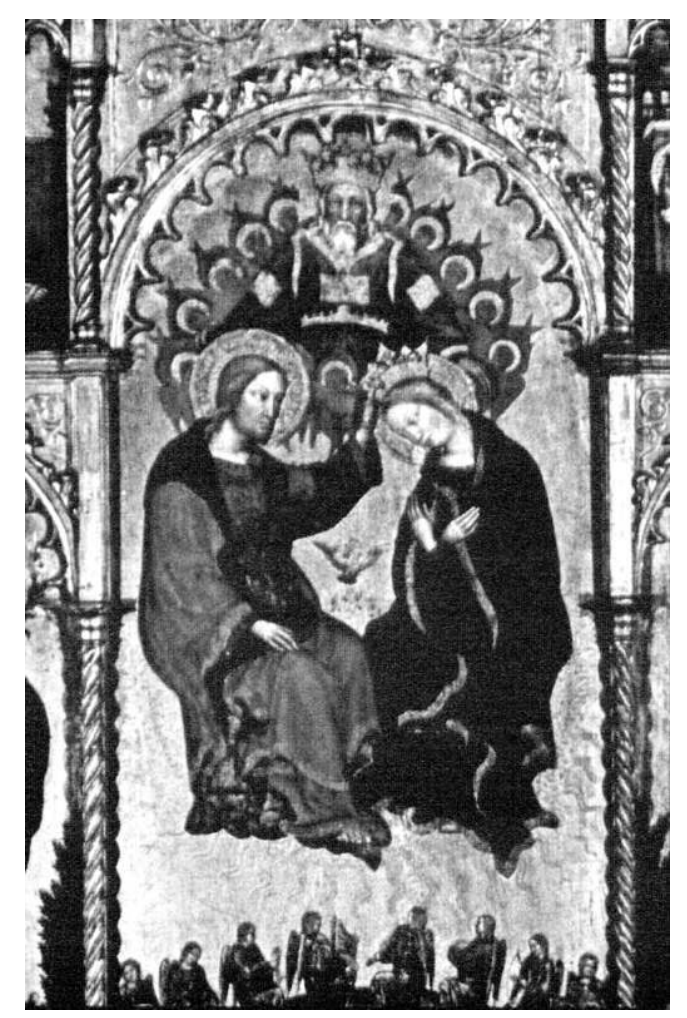

Pinacothèque de Brera

Dans cette représentation du couronnement de la Vierge, les chérubins étagés le long des bras de l'Ancien des Ages ou Verbe Créateur apparaissent comme les notes de l'intervalle de quinte et leurs renversements, alors que les anges musiciens se trouvant dessous figurent les intervalles correspondants. 
Dans le sujet qui nous occupe, il reste une note qui n'a pas encore trouvé d'attribution. C'est la note ré (Rex ou reus, roi ou accusé) qui se trouve par rapport au do comme le sol par rapport au fa, dans un rapport de $9 / 8=3^{2} / 2^{3}$. Le ré est donc associé à l'homme, et une cadence sur le ré après une phrase en fausse modulation, équivaudra à une vision vespérale humaine, donc sujette à la conversion et au repentir, contrairement à celle des anges qui est définitive parce que leur choix a été opéré en toute connaissance de cause.

\section{Quelques types de sonneries}

47 A la lumière de ce qui vient d'être exposé, le fanfares typiques sera aisé à comprendre. Prenons d'abord Le Lancé

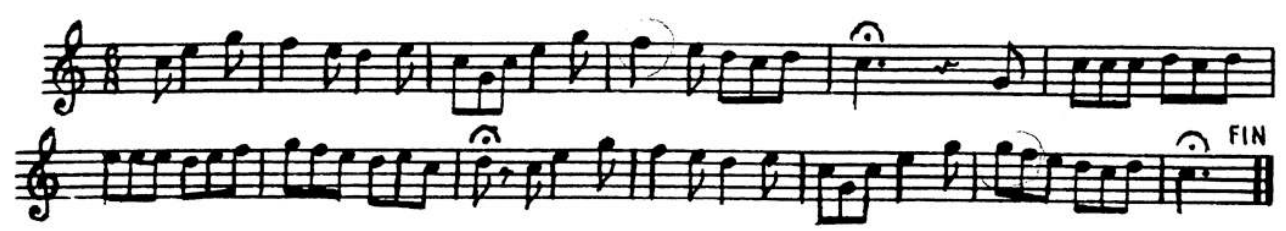

La première phrase à caractère diurnal ne diffère que d'une seule note de la troisième qui est matutinale, et qui redescend ainsi un peu plus rapidement vers la résolution de tonique.

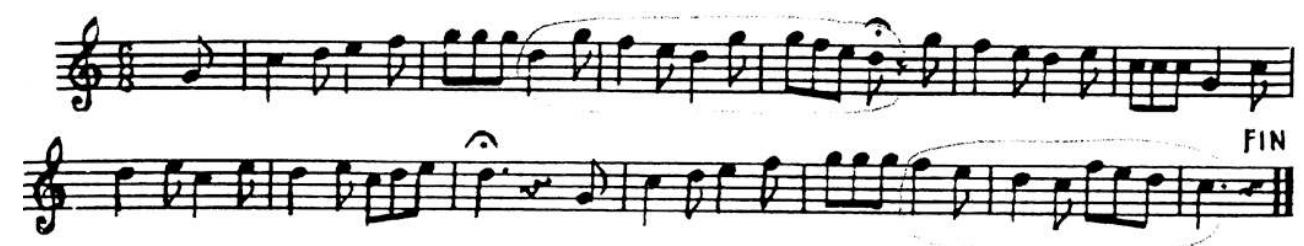

La Vue, qui ne doit être sonnée qu'avec prudence, n'a pas de phrase correspondant à la vision diurnale. La première phrase se résout sur ré sans fausse modulation. Seule la troisième phrase se résout sur la tonique.

Le Lièvre présente une structure légèrement différente. La première vision est figurée par deux phrases, la deuxième par une seule phrase, la troisième à nouveau par deux phrases :

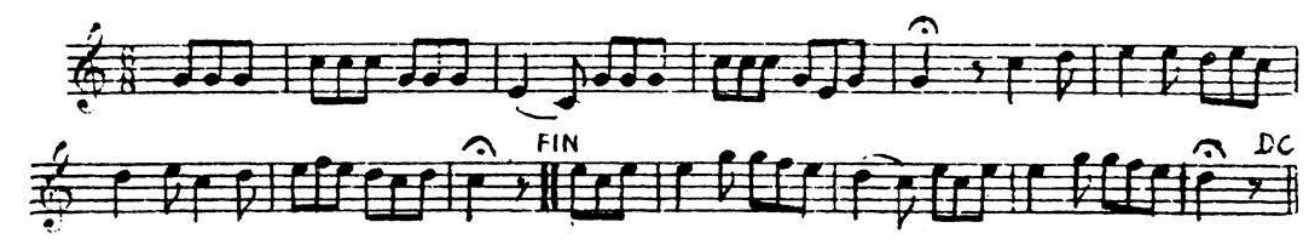

51 La première phrase a une cadence sur sol, la deuxième sur do, ce groupe de phrases correspondant à la vision diurnale, puis vient la vision du soir avec la troisième phrase et sa résolution sur le second degré. Enfin, la conversion est figurée par la répétition des deux premières phrases.

Il existe une version plus ancienne de cette même fanfare : 


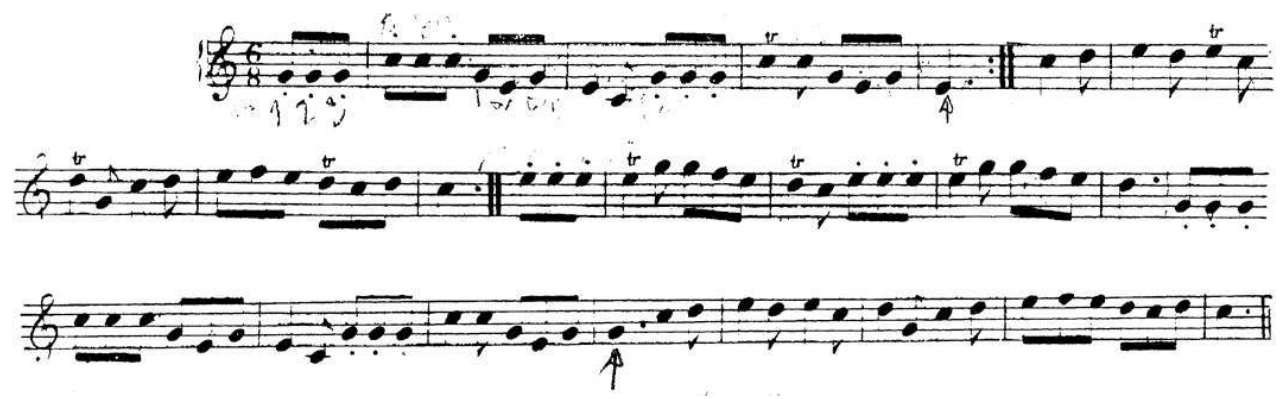

53 Ici, la cadence de la première phrase est faite sur le mi, ce qui fait allusion aux anges. (Il y a un rapport entre mi et mittere, envoyer, et également avec Michael, St Michel l'Archange sonneur de trompe. Le cinquième harmonique, note émotive, est attribuée aux anges) alors que celle de la quatrième est en sol comme dans la version plus récente.

La sonnerie du Débuché, qui se sonne lorsque l'animal sort de la forêt et qu'il est donc possible de le suivre du regard, présente une structure remarquable.

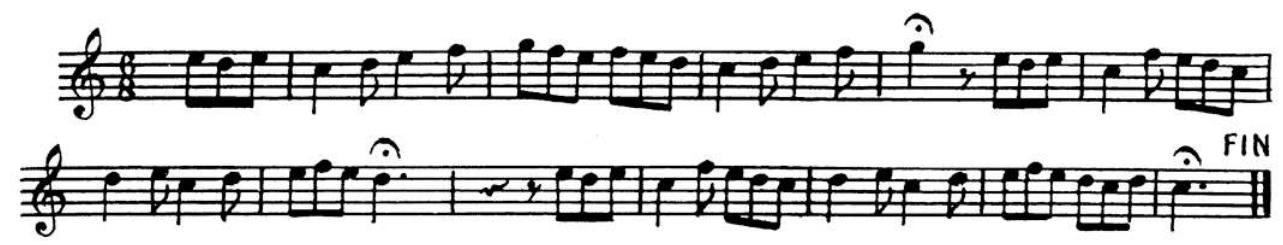

Tous les débuts de phrase sont identiques et les deuxième et troisième phrases ont une importante section commune, ne différant que par la cadence et les quelques notes qui la précèdent.

Enfin, la fanfare des Honneurs, qui se sonne en fin de chasse pour marquer une action d'éclat, comporte six phrases groupées deux par deux, la première de chaque groupe se résolvant sur le second degré, et la seconde sur la tonique, mais les deux phrases du milieu sont en fausse modulation.

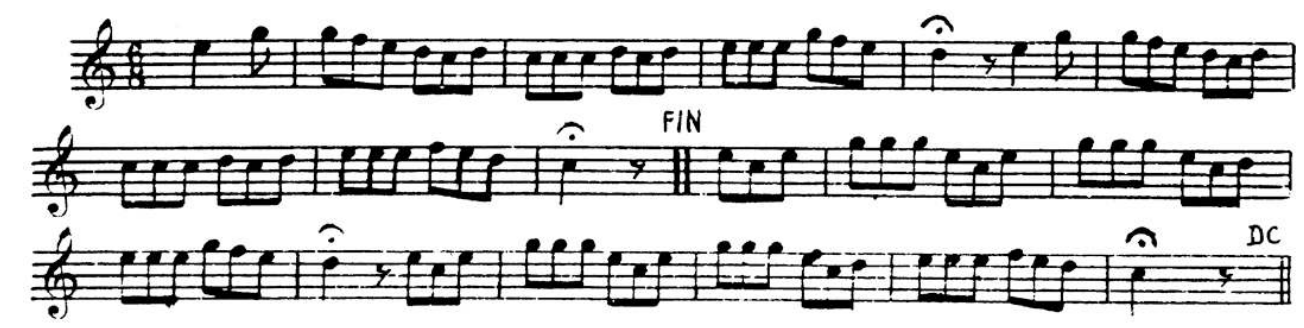

Pour conclure cette brève étude, il convient de répondre à la question suivante : en quoi cette manière de nommer des êtres ou des événements situés en ce monde sensible, si différente du langage ordinaire, peut-elle légitimement être attribuée aux anges et, en fin de compte, de quelle utilité est tout cela?

Il doit être clairement dit qu'une fanfare sonnée à la trompe n'est pas une expression textuelle amenée du monde angélique en ce monde. Par contre, elle peut donner une idée de ce qu'elle serait si elle était parfaitement transportable en ce monde qu'est le nôtre. Disons qu'il y a le même rapport entre la fanfare et le vocable angélique qu'entre notre langage ordinaire et la fanfare. D'autre part, sa structure suggère un autre genre d'étymologie et de syntaxe que la nôtre : c'est l'expression analogique d'une étymologie du Vrai qui se trouve dans le Verbe, lieu des possibles. Dans cette mesure, et dans cette mesure seulement, la pratique musicale traditionnelle est une participation au ministère 
angélique, et en tout cas au ministère adamique, qui consiste à attribuer à toute créature son nom (Genèse 2, 19-20).

On pourrait dire la même chose de la sémantique qui régit le sonner de la trompe, et ceci nous amènerait à examiner de nombreux points importants en musique, dont la gravité n'est plus guère ressentie dans la musique contemporaine, comme l'articulation, la compression, le maintien de la colonne d'air, l'emploi des inégalités rythmiques dans le cadre d'une métrique stricte, ou encore l'usage du vibrato d'intensité, du radouci et l'absence de polyphonie réelle si l'on excepte l'emploi du son bouché. Tous ces éléments se conjuguent dans le choix d'une esthétique qui, autant que l'argument, est objet d'inspiration.

\section{BIBLIOGRAPHIE}

1982, Nouveau recueil de fanfares de chasse de la Fédératlon internationale des trompes de France. Paris.

PHOEBUS Gaston, vers 1387, Le livre de la chasse. Paris : Bibliothèque Nationale. Ch. XVI.

PINGUET Francis, 1978, « La vénerie et sa musique ». La revue musicale. Paris.

SERRE DE RIEUX, 1734, Les dons des enfans de Latone. La musique et la chasse du cerf. Paris.

ANONYME, Vers 1720, Collection de cent airs et fanfares pour la chasse de la maison royale à une ou deux trompes. Paris : Jouve.

SAINT AUGUSTIN, s.d., De musica.

SAINT AUGUSTIN, 1972, De genesi ad litteram. Bibliothèque augustinienne. CEuvres de St Augustin. Traduction, introduction et notes par les Pères Agaese et Solignac. Paris : C.N.R.S. Desclée de Brouwer.

JACQUES DE LIÈGE, 1955, Speculum musicae. Rome : American Institute of Musicology. Livre I particulièrement $\mathrm{Ch}$.

MERSENNE Marin, 1975, Harmonie universelle. Paris : C.N.R.S. Livre V ${ }^{\mathrm{e}}$

\section{ANNEXES}

Saint AUGUSTIN, De Genesi ad Litteram, Bibliothèque augustinienne, Euvres de St Augustin, Traduction, introduction et notes par les Pères Agaese et Solignac, C.N.R.S., Paris : Desclée de Brouwer, 1972. 


\section{Livre quatrième}

\section{Les trois « moments » de la connaissance angelique}

XXII.39. Mais avant la création du ciel appelé firmament dans lequel furent également créés les luminaires, nous ne voyons pas par quel cheminement circulaire ou par quel jeu de progrès et de retrait une lumière corporelle aurait pu produire le jour et la nuit. Il nous semble dès lors que nous ne devons pas laisser la question en suspens sans proposer notre propre hypothèse. Supposons que cette lumière primitivement créée n'est pas corporelle, mais spirituelle : de même qu'elle a succédé aux ténèbres, en ce sens que, à partir d'un stade où elle était encore informe, elle a été formée par conversion vers son créateur, de même le matin succède au soir, lorsqu'après s'être connue en sa propre nature par laquelle elle n'est pas ce qu'est Dieu, elle se rapporte, pour la glorifier, à cette lumière qu'est Dieu lui-même dont la contemplation la fait accéder à sa forme. Et parce que les autres créatures, qui lui sont inférieures, ne sont pas produites sans que cette lumière (spirituelle) en ait connaissance, ce même jour est chaque fois répété : par sa répétition sont produits autant de jours qu'il y a de différents genres de créatures dont la perfection du nombre six doit marquer l'achèvement. Le soir du premier jour serait la connaissance que cette lumière prend aussi d'elle-même, en tant qu'elle n'est pas ce que Dieu est ; le matin, qui suit le soir et qui achève le premier jour pour inaugurer le second, serait le moment où la créature spirituelle se tourne vers le Créateur pour rapporter à sa gloire son être de créature et prendre connaissance, dans le Verbe de Dieu, de la créature qui vient après elle, c'est-à-dire du firmament. Celui-ci est d'abord produit dans la connaissance de cette lumière, quand il est dit : Et il en fut ainsi ; il est ensuite produit dans la nature même du firmament, qui est créé quand l'Ecriture, après avoir déjà dit : Et il en fut ainsi, ajoute : Et Dieu fit le firmament. Ensuite, il y eut un soir pour cette lumière, lorsqu'elle connut le firmament, non plus comme précédemment dans le Verbe de Dieu, mais en sa nature propre : connaissance qui étant inférieure à la précédente, est à juste titre symbolisée par le nom de soir. Après quoi il y eut un matin qui clôt le second iour et commence le troisième. De nouveau ce matin est le moment où cette lumière - c'est-àdire ce jour - se tourne vers Dieu pour le louer d'avoir créé le firmament et pour connaître dans le Verbe la créature qui devait être créée après le firmament. En conséquence, lorsque Dieu dit : Que l'eau qui est sous le ciel s'amasse en un même lieu et qu'apparaisse la terre sèche (Gen.I,9), cette lumière connait cette création dans le Verbe de Dieu par qui cette parole est dite; voilà pourquoi l'Ecriture ajoute : Et il en fut ainsi, c'est-à-dire dans la connaissance que cette lumière en eut dans le Verbe de Dieu. Ensuite, quand l'Ecriture ajoute : Et l'eau fut amassée, etc., alors qu'il a déjà été dit : Et il en fut ainsi, la créature même est créée en son espèce propre. De nouveau, quand cette créature est connue en son espèce propre par cette lumière, qui en avait déjà pris connaissance dans le Verbe de Dieu comme d'une oeuvre à faire, il y eut un troisième soir. Et ainsi de suite pour les autres créations qui se poursuivirent jusqu'au matin qui suivit le soir du sixième jour.

\section{La créature connue dans le Verbe et dans sa nature propre}

XXIII. 40. Il y a une si grande différence entre la connaissance d'une chose dans le Verbe de Dieu et la connaissance de cette chose en sa nature qu'il est légitime d'assimiler l'une au jour, l'autre à la nuit. Car, en comparaison de cette lumière contemplée dans le Verbe 
de Dieu, toute connaissance, par laquelle nous connaissons n'importe quelle créature en elle-même, peut, non sans raison, être qualifiée de nuit. Cette connaissance diffère toutefois tellement de l'erreur ou de l'ignorance de ceux qui ne connaissent pas même la créature en elle-même que, par comparaison, il n'est pas déraisonnable de la qualifier de jour. De même, l'Apôtre n'a pas tort d'appeler lumière et jour la vie que mènent les fidèles en cette chair et en ce monde, par comparaison avec la vie de l'infidèle et de l'impie, lorsqu'il dit : Vous futes jadis ténèbres, maintenant vous êtes lumière dans le Seigneur (Ephes.,V,8), ou encore : Rejetons les œuvres de ténèbres et revêtons les armes de lumière, afin de marcher honnêtement, en plein jour (Rom.,XIII,12,13). Pourtant, si ce jour n'était lui-même une nuit en comparaison de ce jour où, devenus égaux aux anges, nous verrons Dieu tel qu'il est, nous n'aurions pas besoin du flambeau de la prophétie dont l'apôtre Pierre nous dit: " Nous avons foi dans les paroles des prophètes, auxquelles vous faites bien de prêter attention, comme à une lampe en un lieu obscur, jusqu 'à ce que le jour vienne à poindre et que 1 'étoile du matin se lève en nos cœurs (II Pet., I,19).

\section{Connaissance diurnale, matutinale et vespérale ou la triple connaissance angélique}

XXIV. 41. Les saints anges auxquels nous serons égaux après la résurrection, (cf. Matt.,XXII,30) si nous suivons jusqu'au bout la voie - je veux dire le Christ qui s'est fait voie pour nous -, contemplent toujours la face de Dieu et jouissent de la vue du Verbe, son Fils unique, en tant qu'il est égal auPère : c'est en eux, avant toute autre créature, qu'a été créée la sagesse. Il est donc hors de doute qu'ils connaissent l'universalité de la création, où eux-mêmes tiennent la première place, d'abord dans le Verbe de Dieu, comme en celui par qui toutes choses ont été faites, puisqu'en lui sont les raisons éternelles de toutes choses, fussent-elles temporelles ; ensuite dans la créature ellemême, qu'ils connaissent en abaissant en quelque sorte leur regard sur ce qui est au dessous d'eux pour la référer à la gloire du Verbe, car c'est en cette vérité immuable qu'ils voient, comme en leur principe, les raisons d'après lesquelles cette créature a été faite.

Là donc, ils la voient comme dans le jour, d'où procède aussi ce jour premier créé que constitue la très parfaite unité des anges en raison de leur participation à cette même vérité ; ici, ils la voient comme dans le soir. Mais aussitôt il y a un matin (comme cela se vérifie pour chacun des six jours), car la connaissance des anges ne demeure pas dans le créé, mais le rapporte aussitôt à la gloire et à la charité de celui en qui est connue la créature, non comme une chose faite, mais à faire : tant qu'ils se fixent en cette vérité, c'est le jour. Car, si la créature angélique se tournait, fût-ce vers elle-même, et se complaisait davantage en soi qu'en celui dont la participation la béatifie, l'enflure de l'orgueil la ferait déchoir, comme il est arrivé au diable, dont nous parlerons en temps voulu, quand nous aurons à expliquer comment le serpent séduisit l'homme.

\section{Pourquoi il n'est pas parlé de nuit}

XXV. 42. Ainsi donc les anges, tout en connaissant la créature dans cette créature même, préfèrent à ce savoir, par élection et par dilection, le savoir qu'ils en ont dans la Vérité par qui tout a été fait et dont ils deviennent participants : voilà pourquoi, au cours de ces six jours, il n'est pas parlé de nuit, mais, après un soir et un matin, du jour un ; de même, après un soir et un matin, du second jour ; ensuite, après un soir et un matin, du troisième 
jour ; et ainsi de suite jusqu'au matin du sixième jour, à partir duquel commence le septième, jour du repos de Dieu. Bien que ces jours eussent leur nuit, cependant il n'est pas fait mention de nuit dans le récit. Car c'est bien alors que la nuit appartient au jour, non le jour à la nuit, quand ces sublimes et saints anges réfèrent le savoir qu'ils ont de la créature, connue dans la créature même, à l'honneur et à l'amour de celui en qui ils contemplent les raisons éternelles d'après lesquelles elle a été faite. En vertu de cette contemplation parfaitement unanime, ils sont le jour unique que Dieu a fait, auquel l'Eglise elle aussi sera rendue participante, une fois achevé le pèlerinage qu'elle accomplit ici-bas, en sorte que, nous aussi, nous exultions et nous réjouissions en lui (Ps., CVII, 24).

\section{RÉSUMÉS}

Parmi de nombreuses autres caractéristiques, comme l'articulation de consonnes, la rigueur métrique coexistant avec les inégalités, la trompe de vénerie française présente une logique dans l'emploi des cadences qui se réfère aux théories musicales et doctrinales de Boèce et St Augustin : c'est là que, comme dans les peintures du quattrocento italien, le son apparait comme un vocabulaire mis au service de la doctrine chrétienne.

The French hunting horn, apart from other characteristics such as consonantic articulation and metric rigour accompanying inequalities, exhibits a specific rhythmic rationale which refers to the musical theories of Boethius and Saint Augustine. In this case, as in the paintings of fifteenthcentury Italy, sound appears as a vocabulary serving the doctrine of christianism.

\section{AUTEUR}

\section{LUC BRETON}

Luc Breton a commencé très jeune la lutherie qu'il a perfectionné avec plusieurs luthiers et archetiers français. Parallèlement, il a étudié la chimie à l'Ecole polytechnique fédérale de Lausanne. Luthier, spécialisé dans la construction et la restauration des instruments et archets baroques et modernes, il travaille aussi comme chimiste à l'Institut suisse de recherches expérimentales sur le cancer. Il fait de la musicologie et pratique la trompe de chasse dans le contexte de la vénerie française. 